\title{
GRAPHICAL INTERFACE DESIGN FOR WATER PUMPING PROCESS WHICH WORKS WITH A HYDROPHORE
}

\author{
C. R. COSTEA ${ }^{1}$, HELGA SILAGHI ${ }^{1}$, LILIANA MATICA ${ }^{1}$, E. GERGELY ${ }^{1}$, G. HUSI ${ }^{2}$, LAURA COROIU ${ }^{1}$
}

\author{
${ }^{1}$ Department of Control Systems Engineering and Management, \\ University of Oradea, Faculty of Electrical Engineering and Information Technology, \\ Str. Universităţii 1, 410087, Oradea, Romania, \\ E-mail: ccostea@uoradea.ro, hsilaghi@uoradea.ro, lmatica@,uoradea.ro, egergely@uoradea.ro, 1coroiu@uoradea.ro
}

\author{
${ }^{2}$ Department of Electrical Engineering and Mechatronics, \\ University of Debrecen, Hungary, Faculty of Engineering, \\ Egyetem tér 1, H-4032 Debrecen, Hungary, \\ E-mail: husigeza@eng.unideb.hu
}

\begin{abstract}
In this paper, the application of water pumping process is presented. This article presents the design of a human machine interface. Using this interface, it can be possible to track the evolution of pumping water flow. The applications consists of water pumping process simulation. Also, are illustrated and analyzed several cases, depending on the water flow which is inside the tank.
\end{abstract}

Keywords: water pumping, hydrophore, Human Machine Interface, Graphical User Interface.

\section{INTRODUCTION}

The hydrophore is a device for obtaining water from any desired depth.

The hydrophore installation consists of the following elements: electric driving motor, hydrophore tank, water filter, pumps, valve, power supply. In Figure 1 is shown a hydrophore installation.

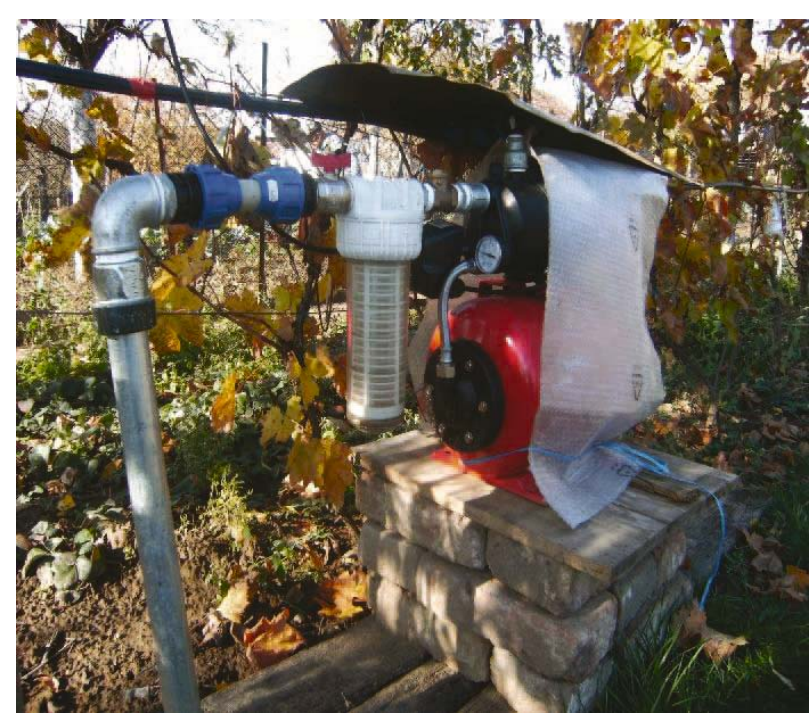

Figure 1. The hydrophore installation

Hydrophore startup is achieved by directly connecting of the motor to mains (to 220 [V]). The hydrophore absorbs water from a reservoir (or under the ground, from a spring) for filling its tank. Primary, in hydrophore tank, the water flow is 0 [liter], likewise 0 [bar] is the air pressure $\{0$ liter, 1 liter, 2 liters $\}$.

From the outside, we can not see the water flow in the hydrophore tank because it has an opaque tank lining; but we can estimate the water flow by knowing the pressure. Using the air gauge of hydrophore, we can see the air pressure inside the network. Pressure is an important parameter because it is an indicator of water flow inside the hydrophore tank (when water flow inside the tank increases, then pressure increases, also and when the flow rate decreases, then pressure decreases).

Air pressure inside matter because only air we can press. Inside the hydrophore tank are both: water and air; a hydrophore tank is never full of water $(100 \%)$, because include water and air.

Considering the case in which hydrophore has a tank of dmax liters, the pressure gauge indicates 0 [bar] when the tank is empty and pmax [bar] when the tank is full. When in the hydrophore tank is water (not mean that necessarily the tank is full), by opening the valve, water flow decreases. Normally, when the pressure reaches at 1 [bar], hydrophore will begin to absorb water until the pressure reaches the pmax [bar] value.

The cycle begins when the pressure drops to 1 [bar]. Then hydrophore will absorb water, that will fill up its tank, until the pressure reaches the pmax [bar] value.

\section{REAL TIME SYSTEM OPERATION}

In the heart of industrial systems, information infrastructure enables innovation, monitors operations, reduces cycle times for production, maintenance and expands market reach [1].

Most engineering is done offline mode. But offline engineering is distinct from real time engineering. The solving method of real time problem, resembles with an artistic event [2].

In the description of control theory, is a big number of words and phrases, that have a large accepted 
significance. The problem of command following appears when the reference is known only at the present time. If the reference is known into the future, then preview is possible and then, the problem is tracking. If unwanted disturbances are applied to the system, then the problem also includes disturbance rejection. There is one aspect of control systems theory, however that apparently does not have an acknowledged name, in particular, the input to the plant [2].

Process control is an essential piece of the water pumping process. The new systems have a digital control system that provides all basic control operations through a computer interface [3].

The controller must connect to human operators, to accept commands from them and to monitor the process status in a quick and understandable form. This is named the human machine interface and is represented in the Figure 2.

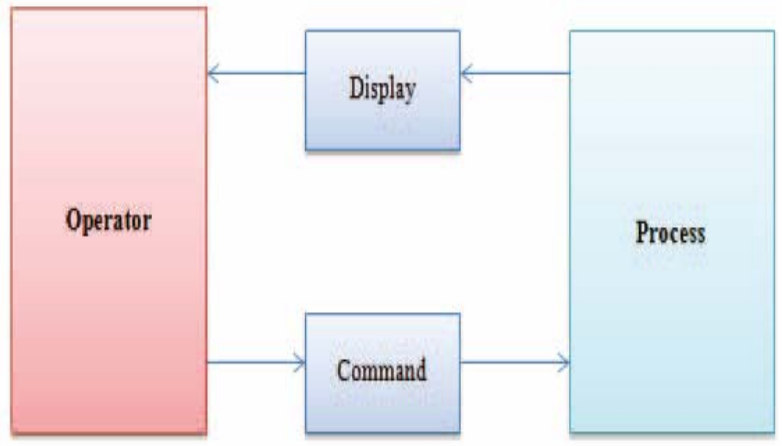

Figure 2. Human machine interface block diagram

\section{DESCRIPTION OF THE GRAPHICAL USER INTERFACE}

To allow process monitoring and operator intervention in the process, if it is necessary, was implemented a GUI (graphical user interface). Thus, the user is able to monitor from the computer, how the water flow is changing. The hydrophore absorbs water from a reservoir and sends it to a receiver.

Graphical user interface was performed using the Visual $\mathrm{I} / \mathrm{O}$ application. Visual I/O is based on objects that have a number of properties that can determine the functioning of graphical objects.

\subsection{Description of Interface Content}

The interface contains two buttons:

- Start button (to start hydrophore, meaning the motor is connected to the mains) is characterized by a boolean variable (with two states: true and false). True state mean that button is pressed and false state means that the button is not pressed. When the button is pressed (condition is true) joined bulb button lights up green. For the situation when the button is not pressed (condition is false) light is off (on the scheme it is gray).
- Open valve button (used to open the valve, which means that water flow will decrease from the hydrophore tank) is characterized by a boolean variable (with two states: true and false). True state mean that mean that button is pressed and false state means that the button is not pressed. When the button is pressed (true state) joined bulb button lights up blue. For the situation when the button is not activated (false state) light is off (on the scheme it is gray).

The graphical user interface has the following elements: on the left is a water reservoir which is the source of the water supply. At this reservoir is linked a pipe. At the other end of this pipe is a pump which is connected to the hydrophore tank (indicated by the horizontal blue tank). On the right side, there is a valve that keep or allow flow the water to go from the hydrophore tank to the recipient (container).

When the application is running, it is posible to illustrate flow of water from tank to the recipient (container). If inside the hydrophore tank is water (water flow in the tank is greater than 0) and the valve opens, the line located between valve and tank container turns blue, which symbolizes the flow of water, in other cases the line will be black (so can be seen by comparing figures 3 and 4).

Also, one can see the numerical values for pressure and for water flow inside the hydrophore tank, both analog and digital form (on display in the center of the scheme) and symbolic form (in two segments - red for pressure and yellow for the flow of water - which is increased or reduced in length along the hydrophore tank).

Under the display in the center of the screen (the one that displays the pressure and water flow) there is a display area for possible alarms.

An alarm situation is when the valve is open and hydrophore tank is empty (water flow $=0$ ). At that time, an alarm appears on the screen (which displays the date and time and the message: 'No water'). If the valve is closed or if hydrophore start to absorbs water (water flow in the tank is greater than 0), then the alarm will close.

\subsection{Description of Interface Running}

When the Start button is pressed (namely, the motor is connected to the mains), the hydrophore absorbs water from a reservoir for filling its tank. Primary, in hydrophore tank, the water flow is 0 [liter], likewise the air pressure is 0 [bar].

If hydrophore is on - water flow from hydrophore tank can be observed through the pressure evolution. This evolution is characterized by the following cycle: from 0 to pmax next to 1 next to pmax next to 1 next to pmax and so on (the pressure increase from 0 bar to the 
maximum value, then when the valve opens, the pressure drops to 1 bar, next hydrophore start to absorb water and the pressure increases again to the pmax value and the procedure is ongoing in the same way until it stops hydrophore).

If hydrophore is off - water flow from the hydrophore tank remain at the same value until the valve opens. If the valve opens, the water flow from the tank starts to drop to 0 (zero liter).

\section{THE SOFTWARE APPLICATION SIMULATION RESULTS}

AND

Some of the operating conditions are illustrated next; those which can be determined from the studies. Therefore an optimal system can be developed [4].

If hydrophore is off and the valve is closed, then the water flow is kept in the hydrophore tank until the valve opening. Hydrophore will fill up after being turned on (Figure 3).

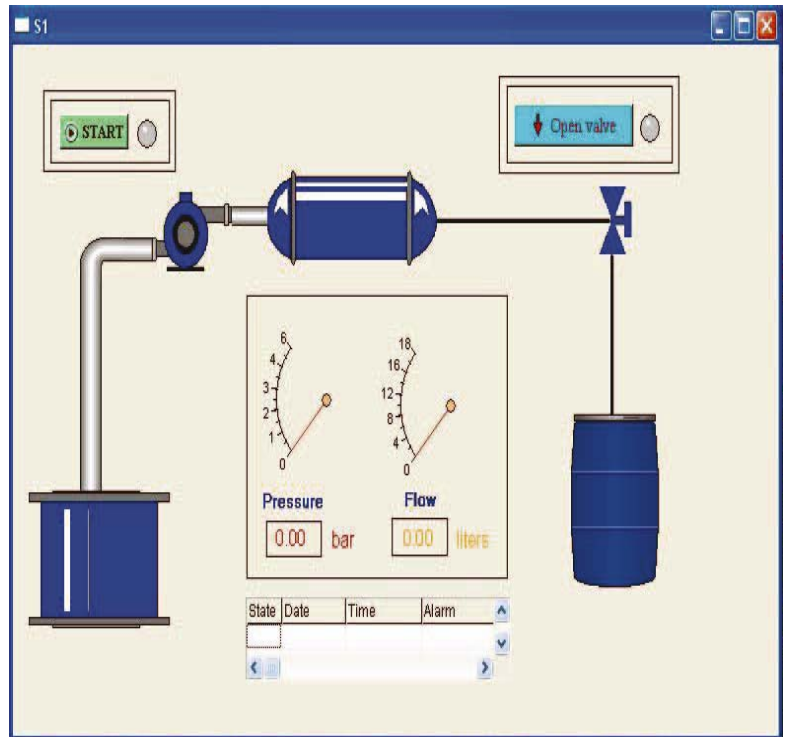

Figure 3. Initial state where both hydrophore, as well as the valve are closed.

If hydrophore is on and valve is closed, the flow will increase until it reaches the dmax value, inside the hydrophore tank, and at the air gauge will see the pressure pmax. Immediately after closing the valve, hydrophore continues to absorbs water until it fill up, therefore until the pressure reaches the maximum value (pmax) (Figure 4).

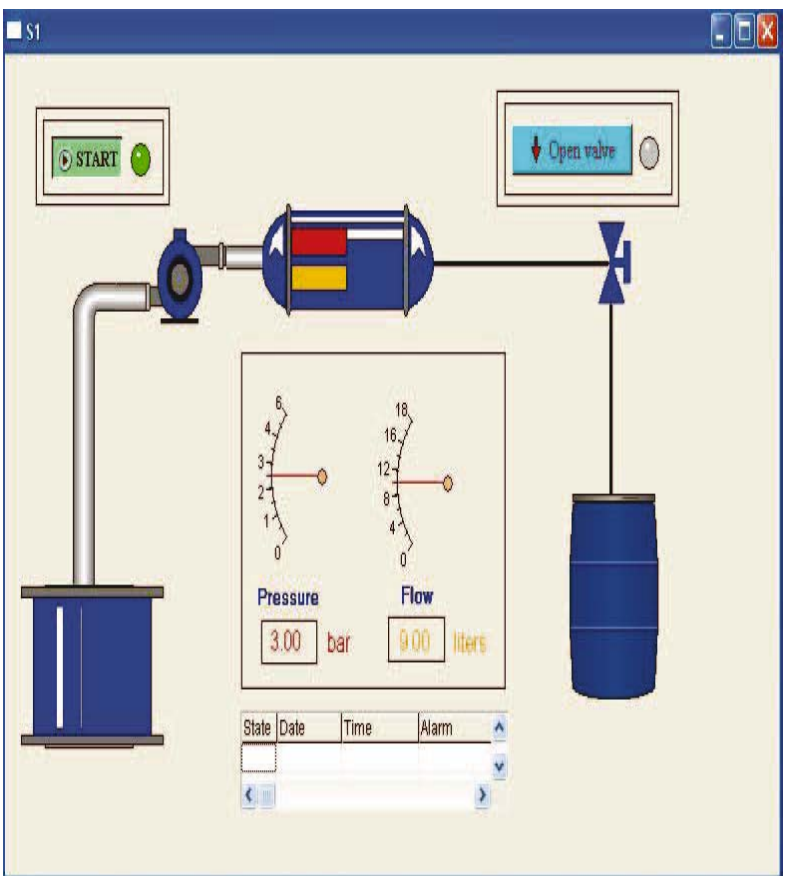

Figure 4. The situation when hydrophore is on and the valve is closed.

If hydrophore is on and valve is open, then water flow from hydrophore tank will decrease until the pressure reaches the value of 1 [bar]. At that time, hydrophore will begin to absorb water and send it, through pipe, to recipient (receiver) (Figure 5).

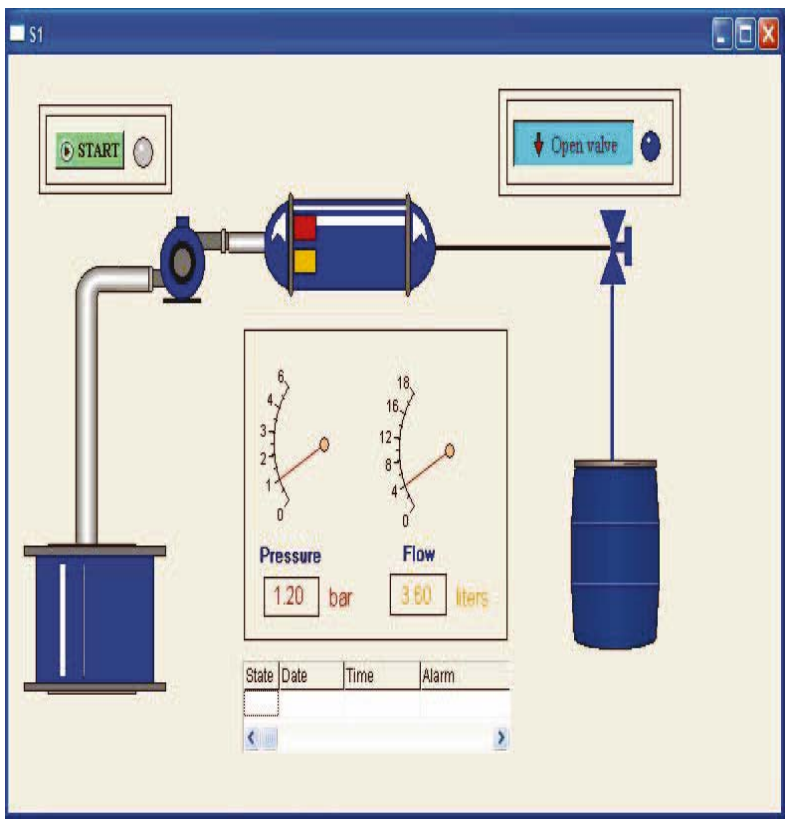

Figure 5. The situation when hydrophore is on and the valve is open.

If hydrophore is off and valve is open, water flow will decrease from hydrophore tank (Figure 6). 


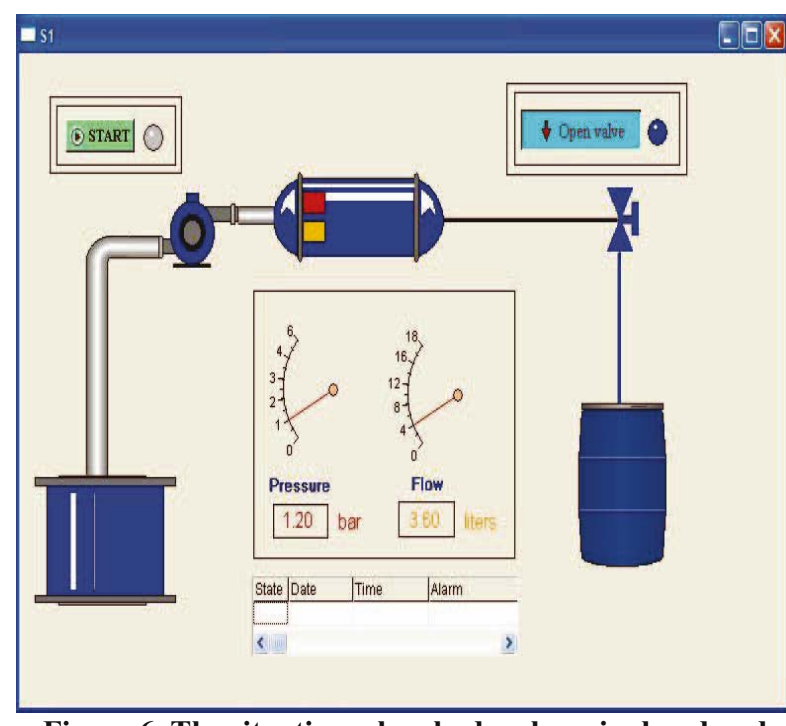

Figure 6. The situation when hydrophore is closed and valve is open (water flow decreases, but there is still water in the tank).

If hydrophore is off and the valve is opened, then water flow will decrease. When it reach at 0 value $(0$ liter and 0 bar) an alarm will appear on screen (which displays the date and time, adding the message: 'No water') (which displays the date and time, but drawing attention to the message: 'No water') (Figure 7).

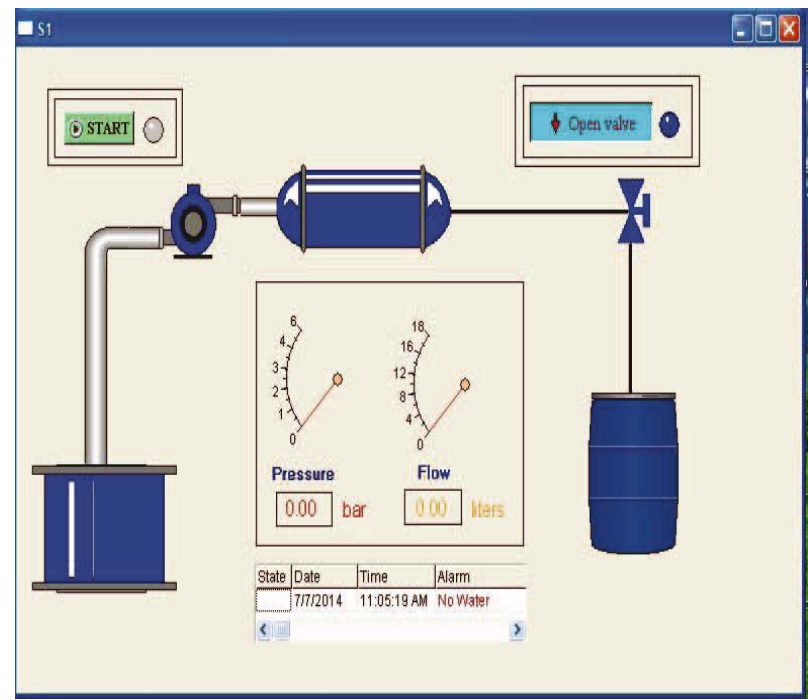

Figure 7. The situation when hydrophore is closed and the valve is opened (occurrence of an alarm because there is no water in the tank).

An optimal design of a human machine interface needs to:

- be designed so as to assist human operators and maintenance staff, when occurs problems or at the preventive maintenance procedures and increase quality in the design procedure approach [5];

- improve operator efficiency to create a design for using human machine interface terminals along the equipment [6];

- reduce the training time, used to learning the operating mode, by the operators, engineers, maintenance staff and supervisors, through congruence on all windows and programs [7];

- allow the operator to provide the required information in an easy to understand format and an easy to use format, so as to allow operators to execute preventive and corrective actions [8-9].

Using a proper Simulink model, it was accomplished the simulation of the process. The simulation results of the process, are shown in the Figure 8.

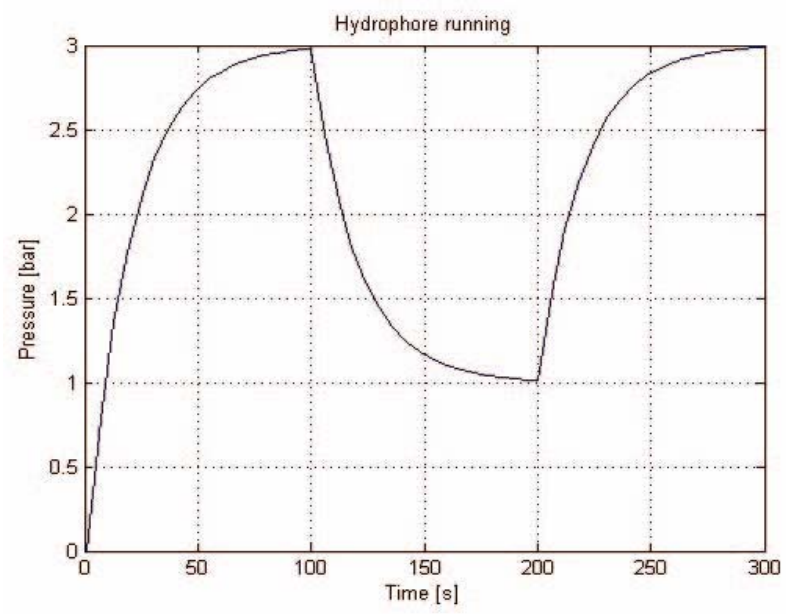

Figure 8. The water flow variation inside the hydrophore tank, during the process.

At baseline (at the simulation start) is considered that the hydrophore tank is empty (the pressure value inside the tank is 0 bar). The water flow evolution from hydrophore tank it can be observed through the pressure evolution.

After the simulation starts, the pressure value increases (because hydrophore absorbs water from a reservoir and so, fills its tank).

The water flow variation is characterized by the following cycle: from 0 to $\operatorname{pmax}$ (i.e., 3 bar), next to 1 bar, next to pmax (i.e., 3 bar), next to 1 bar, next to pmax (i.e., 3 bar). Therefore, the pressure increase from 0 bar to the maximum value, but when the valve opens, then the pressure drops to 1 bar. Further, the hydrophore starts to absorb water, i.e. the pressure is increasing again to the pmax value ( 3 bar) and the procedure is ongoing in the same way until the hydrophore drive is stopped.

\section{CONCLUSIONS}

In this article, we have presented the implementation of a human machine interface. A graphical interface for viewing the evolution of pumping water flow was made. This article describes the interface content and the interface running. Using this interface, it can be possible to track the evolution of pumping water flow. Also, we have presented the process (filling and emptying the hydrophore tank, according to water flow evolution). 
The process has been analyzed through several cases which have been presented.

Finally, were shown some Matlab simulation results. After the process simulation was obtained a plot, which shows the water flow variation in time.

\section{REFERENCES}

[1] M. Capelli-Schellpfeffer, Safety when we will the way, IEEE Industry Applications Magazine, vol. 17, no. 5, ISSN 1077-2618, 2011.

[2] D.S. Bernstein, Rules Rule - Real Time Engineering, IEEE Control Systems Magazine, vol. 31, no. 3, pp. 6-7. 2011

[3] C. R. Costea, H. Silaghi, E.I. Gergely, G. Husi, L. Coroiu, Z. Nagy, Approach of PID Controller Tuning for Ball Mill, Proceedings of 2014 International Symposium on Fundamentals of Electrical Engineering, Bucharest, Romania, November 28-29, 2014, pp. 371-374, 2014.

[4] W.H. Kersting, The whys of distribution system analysis, IEEE Industry Applications Magazine, vol. 17, no. 5, ISSN 1077-2618, pp. 59-65, 2011.
[5] D. Inverso, R. Sokoll, Optimum human-machine interface design, Control Eng. 44(12), pp. 93-98, 1997.

[6] S. Weiss, R. Siegwart, Real-time metric state estimation for modular vision inertial systems, Proceedings of the IEEE International Conference in Robotics Automation, pp. 4531-4537, 2011.

[7] A. Bobtsov, A. Borgul, Human-machine interface for mechatronic devices control, IFAC Proceedings Volumes, ISSN 1474-6670, pp. 614 - 618, 2013.

[8] L. Scott, A. Joseph Torzillo, Human-machine interface systems for production aplications, SMT Surface Mount Technology Magazine, 26(10), pp. 16-30, 2011.

[9] Graham C. Goodwin, Stefan Graebe, Mario E.Salgado, Control System Design. Industrial Applications of Feedforward Control, pp. 227, Valparaiso, Chile, 2000. 